\title{
WIENER INTEGRAL REPRESENTATIONS FOR CERTAIN SEMIGROUPS WHICH HAVE INFINITESIMAL GENERATORS WITH MATRIX COEFFICIENTS
}

\author{
BY DONALD G. BABBITT ${ }^{1}$
}

\author{
Communicated by Richard Arens, January 3, 1967
}

1. Introduction. Let $\mathrm{X}=\left(x_{t}+\infty, M_{t}, P_{x}\right)$ be the Wiener process on $R^{n}$, the real vector space of $n$-tuples. ${ }^{2}$ We assume the process is separable in the sense of Doob so that if $\Omega$ is the sample space for $X$, then $P_{x}\left\{\omega \in \Omega \mid x_{(\cdot)}(\omega)\right.$ is continuous on $\left.[0, \infty)\right\}=1$ for all $x \in R^{n}$. Let $B_{1}, B_{2}, \cdots, B_{n}$, and $V$ be bounded continuous complex $N \times N$ matrix-valued functions on $R^{n}$ and let $\widetilde{D}(B ; V)$ be the closure in $L^{2}\left(\boldsymbol{R}^{n} ; \boldsymbol{C}^{N}\right)$ of the differential operator

$$
D(B ; V)=\frac{1}{2} \sum_{j=1}\left(\frac{\partial}{\partial x^{j}}\right)^{2}+\sum_{j=1}^{n} B_{j}(x) \frac{\partial}{\partial x^{j}}+V(x)
$$

with domain $C_{0}^{\infty}\left(\boldsymbol{R}^{n} ; \boldsymbol{C}^{N}\right)^{1} .\left(C_{0}^{\infty}\left(\boldsymbol{R}^{n}, \boldsymbol{C}^{N}\right)\right.$ denotes the space of infinitely differentiable $C^{N}$-valued functions on $R^{n}$ with compact support.)

It is the purpose of this announcement to state two theorems which prove the existence of an $N \times N$ matrix-valued functional $\alpha_{t}(B ; V)$ on $\Omega \times[0, \infty)$ such that

$$
\left\{T_{t}(P ; V) \phi\right\}(x) \equiv M_{x}\left\{\alpha_{t}(P ; V) \phi\left(x_{t}\right)\right\},
$$

for $\phi \in L^{2}\left(R^{n} ; C^{N}\right)$, defines a strongly continuous semigroup of operators on $L^{2}\left(R^{n} ; C^{N}\right)$ with infinitesimal generator $\widetilde{D}(B ; V)$.

Note that if $-\widetilde{D}(B ; V)$ is the Schrödinger operator for a physical system, perhaps involving internal spin, and $\beta=1 / B T$, then $T_{\beta}(B ; V)$ is the quantum statistical matrix for the system in question. It is this fact which motivated our interest in the problem discussed in this paper. See Ginibre [9] for an interesting discussion of the application of Wiener integral representations to statistical mechanics. ${ }^{3}$

1 Most of the work for this paper was done while the author was on sabbatical leave from the University of California at Los Angeles and in residence at Princeton University. Partial support was also received from NSF Grant 5279.

${ }^{2}$ See [6, Chapter VII]. We shall use the notation introduced by Dynkin without further comment.

${ }^{3}$ The restrictions we have placed on the $\left\{B_{j}\right\}$ and $V$ would only allow us to apply the Wiener integral representation theory to approximations to real situations. It should be remarked that it is not much more difficult to handle the somewhat more realistic case where $V=V_{1}+V_{2}$ and where $V_{1}$ is as above and $V_{2}$ is a real scalarvalued function which is bounded from above on $R^{n}$ and continuous except for a set of capacity zero i.e., when $V_{2}$ is a repulsive potential. 
Much work has been done on Wiener integral representations of diffusion-like semigroups, or, what is the same thing, the Wiener integral representation of the solution of diffusion equations. For example, see Kac [10], Ray [11], Cameron [3], Getoor [8], Daleckii [4], Feldman [7], Beekman [2], the present author [1], and others. Only Daleckii, to the best of our knowledge, considers the case where $N>1$. However his approach is completely different from ours.

For the case $N=1$ and $B_{j} \neq 0$, our results are known but the techniques we use seem to be new and more natural. They are motivated by the techniques Darling and Siegert developed for the case $B_{j} \equiv 0$ for $j=1, \cdots, n$ and $N=1$. For example, see [8].

I would like to thank Professor Edward Nelson for the many conversations we had while we were doing the research for this paper.

2. Main results. We will give the pertinent definitions and state the main results in this section. Proofs will not be given, but the most important lemmas needed in the proofs will be stated. Detailed proofs of the results in this announcement will appear elsewhere.

Notation. If $f$ is a scalar or vector valued on $\Omega$, then $M_{x}\{f\}$ denotes the integral of $f$ with respect to $P_{x}$ if it exists. If $A$ is a complex $N \times N$ matrix, let $|A|^{2}=$ Trace $\left(A A^{*}\right)$ where $A^{*}$ is the complex adjoint of $A$.

Definition. A complex $N \times N$ matrix-valued function $\alpha$ on $\Omega \times[0, \infty)$ is said to be $x$-admissible for $x \in R^{n}$ if $\alpha$ has the following properties:

(i) $\alpha(\cdot, t) \equiv \alpha_{t}(\cdot) \equiv \alpha_{t}$ is $\bar{\eta}_{t}^{x}$-measureable for $t>0$ (see [6, pp. 80-83] for the definition of $\left.\tilde{\eta}_{t}^{x}\right)$;

(ii) $P_{x}\left\{\omega \in \Omega \mid \alpha_{(\cdot)}(\omega)\right.$ is continuous on $\left.[0, \infty)\right\}=1$;

(iii) for each $T>0$,

$$
M_{x}\left\{\int_{0}^{T}\left|\alpha_{t}\right|^{2} \alpha t\right\}<\infty .
$$

Theorem 1. Let $B_{1}, B_{2}, \cdots, B_{n}$, and $V$ be as in $\$ 1$. There then exists a complex $N \times N$ matrix-valued function $\alpha(B ; V) \equiv \alpha$ on $\Omega \times[0, \infty)$ such that:

(a) $\alpha$ is $x$-admissible for all $x \in R^{n}$;

(b) for each $T>0$, there exists a constant $Q_{T}$, independent of $x$, such that

$$
M_{x}\left\{\left|\alpha_{t}\right|^{2}\right\} \leqq Q_{T}
$$

for $0 \leqq t \leqq T$; 
(c) for each $x \in R^{n}$ and $t \geqq 0$,

$$
\alpha_{t}=I+\sum_{j=1}^{n} \int_{0}^{t} \alpha_{\tau} B_{j}\left(x_{\tau}\right) d x_{\tau}^{j}+\int_{0}^{t} \alpha_{\tau} V\left(x_{\tau}\right) d \tau,
$$

almost surely with respect to $P_{x}$ (a.s. $\left.P_{x}\right)$ and where $\sum_{j=1}^{n} \int_{0}^{t} \alpha_{\tau} B_{t}\left(x_{\tau}\right) d x_{\tau}^{j}$ is a stochastic integral and $I$ is the $N \times N$ identity matrix.

See [6, Chapter VII], for a thorough discussion of stochastic integrals.

REMARK. For $N=1$,

$$
\alpha_{t}(\cdot)=\exp \left[\sum_{j=1}^{n} \int_{0}^{t} B_{j}\left(x_{\tau}\right) d x_{\tau}^{j}+\int_{0}^{t}\left(V\left(x_{\tau}\right)-\frac{1}{2} \sum_{j=1}^{n} B_{j}^{2}\left(x_{\tau}\right)\right) d \tau\right]
$$

satisfies (a), (b), and (c) of Theorem 1.

The proof of this theorem follows from the following two lemmas which we state but do not prove.

Lemma 1. Let $B_{1}, B_{2}, \cdots, B_{n}$, and $V$ be as in Theorem 1. Let $x \in R^{n}$. There then exists a complex $N \times N$ matrix-valued function $\alpha^{x}(B ; V) \equiv \alpha^{x}$ on $\Omega \times[0, \infty)$ such that:

( $\left.\mathrm{a}^{\prime}\right) \alpha^{x}$ is $x$-admissible;

$\left(\mathrm{b}^{\prime}\right)$ for each $T>0$, there exists a constant $Q_{T}^{x}$ such that:

$$
M_{x}\left\{\left|\alpha_{t}^{x}\right|^{2}\right\} \leqq Q_{T}^{x}
$$

for $0 \leqq t \leqq T$;

(c') for $t \geqq 0$,

$$
\alpha_{t}^{x}=I+\sum_{j=1}^{n} \int_{0}^{t} \alpha_{\tau}^{x} B\left(x_{\tau}\right) d^{x} x_{\tau}^{j}+\int_{0}^{t} V\left(x_{\tau}\right) d \tau
$$

a.s. $P_{x}$.

Lemma 2. Let $\alpha^{x}, x \in R^{n}$, be the functionals constructed in Lemma 1. There then exists an $x$-admissible functional $\alpha$ on $\Omega \times[0, \infty)$ such that

$$
P_{x}\left\{\alpha_{(\cdot)}^{x}(\omega)=\alpha_{(\cdot)}(\omega) \text { on }[0, \infty)\right\}=1
$$

for all $x \in R^{n}$.

Notation. Let $P(t, x)=(2 \pi)^{-n / 2} \exp \left[-|x|^{2} / 2 t\right]$ where $|x|^{2}$ $=\sum_{j=1}^{n}\left(x^{j}\right)^{2}$. For $\phi \in L^{2}\left(R^{n} ; C^{N}\right)$, let

$$
\left(T_{t} \phi\right)(x) \equiv \int_{R^{n}} P(t, x-y) \phi(y) d y .
$$


It is well known that $T_{t}$ is a strongly continuous semigroup of operators on $L^{2}\left(\boldsymbol{R}^{n} ; \boldsymbol{C}^{N}\right)$ with infinitesimal generator $\widetilde{D}(0 ; 0)$ (see $\$ 1$ ).

TheOREM 2. Let $B_{1}, B_{2}, \cdots, B_{n}, V_{1}$ and $\alpha(B ; V) \equiv \alpha$ be as in Theorem 1. Then for each $\phi \in L^{2}\left(\boldsymbol{R}^{n} ; C^{N}\right), x \in R^{n}$, and $t \geqq 0$,

$$
\left(T_{t}(B ; V) \phi\right)(x) \equiv M_{x}\left\{\alpha_{t} \cdot \phi\left(x_{t}\right)\right\}
$$

exists and defines an element of $L^{2}\left(R^{n} ; C^{N}\right)$. Moreover $\left\{T_{t}(B ; V): t \geqq 0\right\}$ is a strongly continuous semigroup of operators on $L^{2}\left(\boldsymbol{R}^{n} ; C^{N}\right)$ with infinitesimal generator $\widetilde{D}(B ; V)$.

The main step in the proof of this theorem is to prove the following lemma.

Lemma 3. Let $C_{0}\left(\boldsymbol{R}^{n} ; \boldsymbol{C}^{N}\right)$ denote the continuous $\boldsymbol{C}^{N}$-valued functions on $R^{n}$ with compact support. Then for all $t>0, x \in R^{n}$, and $\phi \in C_{0}\left(\boldsymbol{R}^{n} ; \boldsymbol{C}^{N}\right)$ we have:

$$
\left(T_{t}(B ; V) \phi\right)(x)=\left(T_{t} \phi\right)(x)+\int_{0}^{t}\left(T_{\tau}(B ; V)\left[P T_{t-\tau} \phi\right](x) \cdot d \tau\right.
$$

where $P=\sum_{j=1}^{n} B_{j}(\cdot) \partial / \partial x^{j}+V(\cdot)$.

\section{BIBLIOGRAPHY}

1. D. Babbitt, The Wiener integral and the Schrödinger operator, Trans. Amer. Math. Soc. 116 (1965), 66-78. See also Correction to the Wiener integral and the Schrodinger operator, ibid., 121 (1966), 549-552.

2. J. A. Beekman, Gaussian processes and generalized Schrödinger equations, J. Math. Mech. 14 (1965), 789-806.

3. R. H. Cameron, The generalized heat equation and a corresponding Poisson formula, Ann. of Math. 50 (1954), 434-462.

4. Yu. L. Daleckii, Continual integrals associated with certain differential equations and systems, Soviet Math. Dok1. 2 (1961), 259-263.

5. N. Dunford and J. Schwartz, Linear operators, Part I, Wiley and Interscience, New York, 1958.

6. E. B. Dynkin, Markov processes, Vol. 1, Academic Press, New York, 1965.

7. J. Feldman, On the Schrödinger and heat equations with non-negative potentials, Trans. Amer. Math. Soc. 108 (1963), 251-264.

8. R. K. Getoor, Additive functionals of a Markov process, Pacific J. Math. 7 (1957), 1577-1591.

9. J. Ginibre, Reduced density matrices of quantum gases. I, II, and III, J. Math. Phys. 6 (1965), 238-251, 252-262, 1432-1446.

10. M. Kac, On some connections between probability theory and differential and integral equations, Proc. Second Sympos. on Mathematical Statistics and Probability, pp. 184-215, Univ. of Calif. Press, Berkeley, Calif., 1951.

11. D. Ray, On the spectra of second order differential operators, Trans. Amer. Math. Soc. 77 (1954), 299-321.

Princeton University 